\title{
ON TWO CONJECTURES CONCERNING THE PARTIAL SUMS OF THE HARMONIC SERIES
}

\author{
STEPHEN M. ZEMYAN
}

\begin{abstract}
Let $S_{n}$ denote the $n$th partial sum of the harmonic series. For a given positive integer $k>1$, there exists a unique integer $n_{k}$ such that $S_{n_{k}-1}<k<S_{n_{k}}$. It has been conjectured that $n_{k}$ is equal to the integer nearest $e^{k-\gamma}$, where $\gamma$ is Euler's constant. We provide an estimate on $n_{k}$ which suggests that this conjecture may have to be modified. We also propose a conjecture concerning the amount by which $S_{n_{k}-1}$ and $S_{n_{k}}$ differ from $k$.
\end{abstract}

\section{The first conjecture. Let}

$$
S_{n}=\sum_{m=1}^{n} \frac{1}{m}
$$

denote the $n$th partial sum of the harmonic series. It is a well-known fact (see [7, pp. $380-381$ ]) that $S_{n}$ is never an integer for $n>1$. Since the harmonic series diverges, there exists for each integer $k>1$ a corresponding integer $n_{k}$ such that

$$
S_{n_{k}-1}<k<S_{n_{k}} \text {. }
$$

The question we wish to address here is: What is the value of $n_{k}$ for each $k>1$ ? It has been conjectured in Advanced Problems 5346 (1967, p. 209) and 5989 (1974, p. $910)$ in the American Mathematical Monthly that $n_{k}$ is equal to $\left(\left(e^{k-\gamma}\right)\right)$, where $\gamma=0.5772156649 \cdots$ is the Euler-Mascheroni constant and $((x))$ denotes the integer nearest $x$. L. Comtet [6, p. 209] shows that either $n_{k}=\left[e^{k-\gamma}\right]$ or $\left[e^{k-\gamma}\right]+1$, where $[x]$ denotes the greatest integer $\leq x$. R. P. Boas comments $[2$, p. 749] in a partial solution to this problem that this conjecture has been verified by computation for $k \leq 200$ by Boas (unpublished) and for $k \leq 1000$ by R. Spira (unpublished). Boas also establishes [5, p. 865] the following partial result: If $m$ and $\delta$ are the integral and fractional parts of $e^{k-\gamma}$, then $n_{k}=\left(\left(e^{k-\gamma}\right)\right)$ provided that $\delta \notin[1 / 2-$ $1 / 10 m, 1 / 2+1 / m]$.

The following result suggests that the above conjecture may possibly be improved.

THEOREM. For each integer $k>1$, the integer $n_{k}$ satisfies the inequality

$$
-\frac{2}{e^{3(k-\gamma)}}-\frac{1}{2}<n_{k}-\left(e^{k-\gamma}-\frac{1}{24 e^{k-\gamma}}\right)<+\frac{1}{2}+\frac{2}{e^{3(k-\gamma)}} \text {. }
$$

Received by the editors June 6, 1984 and, in revised form, October 15, 1984. Presented to the Society at the 90th Annual Meeting in Louisville, Kentucky on January 25, 1984.

1980 Mathematics Subject Classification. Primary 40A05. 
REMARK. This theorem suggests that $n_{k}$ may be equal to the integer nearest $e^{k-\gamma}-1 /\left(24 e^{k-\gamma}\right)$. On one hand, the original conjecture could perhaps be modified to reflect the new information contained in the above theorem. On the other hand, this theorem also suggests that we are not yet in a position to postulate a "correct" conjecture as to the value of $n_{k}$.

Before proving this theorem, we digress in order to pose the following question: Is it true that

$$
\left(\left(e^{k-\gamma}\right)\right)=\left(\left(e^{k-\gamma}-\frac{1}{24 e^{k-\gamma}}\right)\right)
$$

for every integer $k>1$ ? More generally, for which positive integers $N$ and real numbers $\alpha$ is it true that

$$
\left(\left(e^{k-\alpha}\right)\right)=\left(\left(e^{k-\alpha}-\frac{1}{N e^{k-\alpha}}\right)\right)
$$

for all integers $k>1$ ? If (5) holds for some fixed $N$ and all $k>1$, must $\alpha$ be transcendental? Let $A$ denote the set of all $\alpha$ for which (5) holds for some fixed integer $N$ and all integers $k>1$. Is the Lebesgue measure of $A$ equal to zero?

Proof OF THE THEOREM. We first establish some background information. It is well known that

$$
\lim _{n \rightarrow \infty}\left(\sum_{m=1}^{n} \frac{1}{m}-\ln n\right)=\gamma
$$

If we define, for $n \geq 1$,

$$
\delta(n)=\sum_{m=1}^{n} \frac{1}{m}-\ln n-\gamma
$$

then it is easily shown that $\delta(n) \downarrow 0$ as $n \rightarrow \infty$. An application of the EulerMaclaurin Summation Formula (see [1, p. 444, 4, pp. 261-262 or 3, p. 256]) yields the estimate

$$
\delta(n)=1 /(2 n)-1 /\left(12 n^{2}\right)+R_{n}
$$

where $0<R_{n}<1 /\left(120 n^{4}\right)$.

Now define the rational number $r_{k}$ by the relation

$$
k=1+\frac{1}{2}+\cdots+\frac{1}{n_{k}-1}+\frac{r_{k}}{n_{k}} .
$$

Note especially that $0<r_{k}<1$. Using (7) and (9), we obtain the relation

$$
\ln n_{k}=k-\gamma-\delta\left(n_{k}\right)+\left(1-r_{k}\right) / n_{k}
$$

which may be exponentiated to obtain $n_{k}=e^{k-\gamma} e^{\beta\left(n_{k}\right)}$, where we have set

$$
\beta\left(n_{k}\right)=-\delta\left(n_{k}\right)+\frac{1-r_{k}}{n_{k}}=\left(\frac{1}{2}-r_{k}\right) \frac{1}{n_{k}}+\frac{1}{12 n_{k}^{2}}-R_{n_{k}} .
$$

Consequently, we have

$$
n_{h}-e^{h-\gamma}=e^{h-\gamma}\left(e^{\left.\beta_{(} n_{h}\right)}-1\right) .
$$


We first show that

$$
-1<n_{k}-e^{k-\gamma}<+1 .
$$

Since $\delta(n)>0$ for all $n \geq 1$, we have

$$
\ln \left(n_{k}-1\right)<\ln \left(n_{k}-1\right)+\delta\left(n_{k}-1\right)=S_{n_{k}-1}-\gamma<k-\gamma .
$$

Exponentiating, we obtain the right-hand side of (13). Since $\ln (n+1)-\ln (n)>$ $1 /(n+1)>\delta(n)$ for all $n \geq 1$, we have

$$
\ln \left(n_{k}+1\right)>\ln \left(n_{k}\right)+\delta\left(n_{k}\right)=S_{n_{k}}-\gamma>k-\gamma .
$$

Exponentiating, we obtain the left-hand side of (13).

Now let $n_{k}-e^{k-\gamma}=a$. From (12) we obtain the equation

$$
\beta\left(n_{k}\right)=\ln \left(1+\frac{a}{e^{k-\gamma}}\right) .
$$

Substituting (11) into (14) and solving for $r_{k}$, we get

$$
r_{k}=\frac{1}{2}+\frac{1}{12 n_{k}}-n_{k} R_{n_{k}}-a \ln \left(1+\frac{a}{e^{k-\gamma}}\right)-e^{k-\gamma} \ln \left(1+\frac{a}{e^{k-\gamma}}\right) .
$$

Now, since $|a|<1$, we may write

$$
-a \ln \left(1+\frac{a}{e^{k-\gamma}}\right)=-\frac{a^{2}}{e^{k-\gamma}}+\frac{a^{3}}{2 e^{2(k-\gamma)}}+\frac{c_{1}}{e^{3(k-\gamma)}},
$$

where $\left|c_{1}\right| \leq 4 / 9$, and

$$
-e^{k-\gamma} \ln \left(1+\frac{a}{e^{k-\gamma}}\right)=-a+\frac{a^{2}}{2 e^{k-\gamma}}-\frac{a^{3}}{3 e^{2(k-\gamma)}}+\frac{c_{2}}{e^{3(k-\gamma)}}
$$

where $\left|c_{2}\right| \leq 1 / 3$. Also,

$$
\frac{1}{12 n_{k}}=\frac{1}{12 e^{k-\gamma}}-\frac{a}{12 e^{2(k-\gamma)}}+\frac{c_{3}}{e^{3(k-\gamma)}}
$$

where $\left|c_{3}\right| \leq 1 / 9$, and

$$
0<n_{k} R_{n_{k}}<\frac{1}{120 n_{k}^{3}}<\frac{1}{9 e^{3(k-\gamma)}} .
$$

The bounds on $c_{1}, c_{2}$ and $c_{3}$ are independent of $k$. Hence,

$$
r_{k}=\left(\frac{1}{2}+\frac{1}{12 e^{k-\gamma}}+\frac{p}{e^{3(k-\gamma)}}\right)-a\left(1+\frac{1}{12 e^{2(k-\gamma)}}\right)-\frac{a^{2}}{2 e^{k-\gamma}}+\frac{a^{3}}{6 e^{2(k-\gamma)}}
$$

where $|p|<1$. This last expression is a polynomial in $a$ from which the theorem is easily deduced using the inequality $0<r_{k}<1$.

2. A second conjecture. Let us again consider inequality (2) and ask the following question: By how much must the closest partial sums miss a given integer $k$ ? More precisely, can one determine functions $\alpha$ and $\beta$ of $k$ alone such that

$$
S_{n_{k}}-k \geq \alpha(k)
$$

and

$$
k-S_{n_{k}-1} \geq \beta(k) ?
$$


Recalling equation (9), we get

$$
S_{n_{k}}-k=\left(1-r_{k}\right) / n_{k}
$$

and

$$
k-S_{n_{k}-1}=r_{k} / n_{k} .
$$

Thus, any lower estimate of the differences in (17) or (18) will involve estimates of $r_{k}$ and $n_{k}$.

Suppose now that the original conjecture about the value of $n_{k}$ is true; i.e., that $\left|n_{k}-e^{k-\gamma}\right|=|a|<1 / 2$. (It was noted earlier in this paper that the conjecture is true for values of $k \leq 1000$.) If $a>-1 / 2$, then equation (16) implies that

$$
1-r_{k}>\frac{1}{24 e^{k-\gamma}}+\frac{1}{16 e^{2(k-\gamma)}}+\frac{p}{e^{3(k-\gamma)}},
$$

where $|p|<1$. Combining (15), (17), (19) and (21), we hypothesize that we may choose

$$
\alpha(k)=1 / 24 e^{2(k-\gamma)}+1 / 48 e^{3(k-\gamma)}
$$

and that $1 / 24$ is the best possible constant here. The author is unable at this time to propose an analogous hypothesis concerning the order of magnitude of $\beta(k)$, but it seems reasonable to suggest that $\beta(k)=O\left(e^{-2 k}\right)$.

\section{REFERENCES}

1. T. M. Apostol, Calculus, Blaisdell, New York, 1962.

2. R. P. Boas, Partial solution to advanced problem 5989*, Amer. Math. Monthly 83 (1976), 749.

3. , Partial sums of infinite series, and how they grow, Amer. Miath. Monthly 84 (1977), 237-258.

4. , Growth of partial sums of divergent series, Math. Comp. 31 (1977), 257-264.

5. R. P. Boas and J. W. Wrench, Jr., Partial sums of the harmonic series, Amer. Math. Monthly 78 (1971), 864-870.

6. L. Comtet, Problem 5346, Amer. Math. Monthly 74 (1967), 209.

7. G. Polya and G. Szegö, Aufgaben und Lehrsätze aus der Analysis, vol. 2, Springer-Verlag, Berlin, 19?5, pp. 159, 380-381; problems VII, pp. 250, 251.

Department of Mathematics, The Pennsylvania State Univeristy, Mont Alto, Pennsylvania 17237 